\title{
Statistical features analysis of transient current signal for broken bars fault detection in
} LS-PMSMs

\begin{abstract}
Electrical motors constitute critical elements in certain industrial facilities and domestic fields as a prime mover. In spite of its good trait, failures in electrical motor cannot be impeded because it is mainly working in the industrial field. LS-PMSM is a high efficiency motor introduced recently in industrial field. LS-PMSM is a hybrid motor, which its rotor is combination of squirrel-cage rotor and high energy permanent magnets to have high starting torque and high efficiency together. Similar to other types of electrical motors, various faults that lead to malfunction of the motor occur in LS-PMSM during its operation. In this context, investigates diagnostic techniques for LS-PMSM with special reference to rotor faults. Since LS-PMSM has squirrel cage rotor, among different faults may happen in it, broken bar is the most important one. This paper deal with the finite element method to investigation of broken rotor bar faults on LS-PMSM with statistical feature analysis in the time domain using transient current signal.
\end{abstract}

Keyword: Broken rotor bar fault; LS-PMSM; Statistical feature; Time domain; Transient current signa 